Hugo R. Zuleta

\begin{abstract}
ANÁLISIS LÓGICO-SEMÁNTICO
DE LAS NORMAS JURÍDICAS:

LAS NORMAS COMO CONTEXTOS

NO EXTENSIONALES EN

EL RAZONAMIENTO JURÍDICO
\end{abstract}




\title{
ANÁLISIS LÓGICO-SEMÁNTICO DE LAS NORMAS JURÍDICAS: LAS NORMAS COMO CONTEXTOS NO EXTENSIONALES EN EL RAZONAMIENTO JURÍDICO
}

\author{
Hugo R. Zuleta \\ Universidad de Buenos Aires
}

\section{Las normas generales como condicionales}

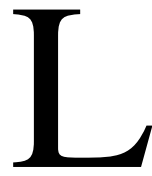

as normas jurídicas generales pueden ser concebidas como enunciados que atribuyen determinadas consecuencias normativas a la presencia de ciertas circunstancias fácticas que se describen genéricamente mediante la mención de propiedades.

La interpretación de la estructura lógica de este tipo de normas presenta considerables dificultades. A primera vista parece razonable concebirlas como condicionales materiales cuyo antecedente hace referencia a algún tipo de evento, hecho, acción o estado de cosas, y cuyo consecuente menciona una acción genérica caracterizada deónticamente. Sin embargo, esto acarrea el inconveniente de que se puede obtener fácilmente, a partir de normas que se refieren a situaciones identificadas a través de propiedades lógicamente independientes, normas que vinculan las mismas circunstancias con exigencias incompatibles.

Por ejemplo, si la norma 1 establece: $p \rightarrow O A$ y la norma $2: q \rightarrow O\ulcorner A$, se puede inferir de la primera, en virtud de la ley de refuerzo del antecedente, la norma 1': (p.q) $\rightarrow O A$, mientras que la segunda implica, por la misma ley, la norma $2^{\prime}:(p . q) \rightarrow O\ulcorner A$. De acuerdo con la terminología utilizada por Alchourrón y Bulygin (1974), diríamos que las normas $1^{\prime}$ y $2^{\prime}$ relacionan el mismo caso genérico, esto es, el definido por la presencia conjunta de las propiedades $p$ y $q$, con soluciones incompatibles, lo que constituye una contradicción normativa.

Cabe advertir que en la obra citada las normas no son tratadas explícitamente como condicionales, de modo que no existe referencia explícita a la ley de refuerzo del antecedente. Sin embargo, esta ley opera a través de las relaciones entre universos de casos de distintos niveles (ver cap. VI, apartado 1), de modo que el tipo de contradicción indicada anteriormente aparece al pasar de los universos de casos definidos respectivamente por las propiedades $p$ y $q-\mathrm{UC}(p)$ y $\mathrm{UC}(q)$ - a un universo de casos más fino, definido mediante ambas propiedades $-\mathrm{UC}(p q)$-. 
En un trabajo posterior, Alchourrón (1991) reconoce que la consecuencia apuntada no refleja adecuadamente, «en la mayoría de los casos», el modo de razonar de los juristas ${ }^{1}$, y analiza tres formas de reconstruir las intuiciones de éstos:

(a) reformular el sistema considerando a una de las normas como una excepción a lo dispuesto por la otra;

(b) sostener que las normas condicionales sólo especifican deberes prima facie, que pueden resultar cancelados al presentarse ciertas circunstancias no contempladas en la norma -lo que importa renunciar tanto a la ley de refuerzo del antecedente como al modus ponens-, y

(c) establecer una relación jerárquica entre las normas, haciendo prevalecer una de ellas en las situaciones conflictivas.

Cada uno de estos procedimientos presenta algunos inconvenientes, como muestra Alchourrón convincentemente.

Sin embargo, el punto que me interesa destacar aquí es otro: que todos ellos tienen por objeto solucionar un real conflicto de normas. En efecto, en el ejemplo que presenta Alchourrón las normas son incompatibles, aunque las intuiciones de los juristas indiquen lo contrario, pues en los casos en que están presentes conjuntamente las propiedades mencionadas en los antecedentes de ambas normas sólo es posible aplicar una de ellas ${ }^{2}$.

\section{Problemas persistentes}

A mi modo de ver hay cierto tipo de normas cuyo tratamiento por cualquiera de los procedimientos indicados anteriormente no logra reconstruir correctamente el razonamiento corriente de los juristas.

Ello es así porque una reconstrucción adecuada debería, en mi criterio, mostrar que las exigencias contenidas en las normas no son en realidad incompatibles, sino que todas son simultáneamente aplicables en los casos en que concurren las propiedades contempladas en los antecedentes de ambas.

Para mostrar el tipo de problema que deseo considerar tomaré ejemplos pertenecientes al campo del derecho tributario, donde coexisten normas que establecen la obligación de pagar impuestos cuando se realizan actividades

${ }^{1}$ Tratando un ejemplo en el que se produce una contradicción de este tipo (ver nota 2) dice Alchourrón: «Sin embargo, en la mayoría de los casos los juristas dirían que las normas en cuestión no establecen en absoluto conflictos de obligación» (p. 267).

${ }^{2}$ El ejemplo de Alchourrón consiste en el siguiente par de normas: «(1) Los jueces deben castigar a los que han cometido homicidio. (2) Los jueces no deben castigar a los menores de edad» (p. 267). Es evidente que en el caso de un homicidio cometido por un menor de edad no es posible aplicar ambas normas. La razón por la que los juristas no advierten el conflicto probablemente sea que dan por sentado, como si se tratara de una necesidad lógica, que la segunda prevalece sobre la primera. 
de cierto tipo con otras que prevén la permisión de no pagar impuestos en ciertos casos.

Consideremos el sistema integrado por las siguientes normas:

Norma 1: Si alguien realiza una actividad onerosa habitual en el territorio de la Provincia de Buenos Aires debe pagar impuestos (por realizar esa actividad).

Norma 2: Si alguien transita de una provincia a otra, le está permitido no pagar impuestos (por transitar el territorio).

Ignoremos, por el momento, las cláusulas colocadas entre paréntesis. Tenemos, entonces, que hay una acción genérica, la de pagar impuestos, que está caracterizada deónticamente de distinta manera en ambas normas.

$\mathrm{Si}$ concebimos a las normas como condicionales materiales, encontramos que la norma 1 posee la estructura $« p \rightarrow O A »$, y la norma 2 , la estructura $« q \rightarrow P\ulcorner A »$. Como se vio en el apartado anterior, mediante la ley de refuerzo del antecedente se puede inferir de la primera la norma « $(p . q) \rightarrow O A »$, y de la segunda, «(p.q) $\rightarrow P\ulcorner A »$, con lo que obtenemos un sistema inconsistente.

Sin embargo, un jurista probablemente afirmaría que no existe inconsistencia alguna porque se trata de impuestos distintos. En efecto, como indican las cláusulas colocadas entre paréntesis, la norma 1 establece un impuesto por la realización de una actividad onerosa en forma habitual, mientras que la exención prevista en la norma 2 se refiere a la actividad de transitar el territorio, que es conceptualmente distinta.

La dificultad que me interesa destacar aparece cuando intentamos reconstruir adecuadamente esta idea con el aparato lógico de que disponemos.

Supongamos que una persona realiza una actividad onerosa habitual en el territorio de la provincia de Buenos Aires y que ésta consiste en trasladar pasajeros o mercaderías a otras provincias. ¿Debe pagar el impuesto establecido en la norma 1 o su actividad está alcanzada por la exención prevista en la norma 2? Asumiré que la respuesta que se desea justificar es que debe pagar el impuesto por la actividad onerosa habitual y que esto no acarrera conflicto alguno con la norma 2, porque la actividad por la que tributa es conceptualmente distinta de aquélla por la que está exento, aunque en el caso sean llevadas a cabo a través de las mismas operaciones observables ${ }^{3}$.

Para que pueda apreciarse con mayor claridad la intuición cuya reconstrucción me interesa presentaré un ejemplo en el que lo que está en juego no es la idea de contradicción sino la de redundancia normativa:

${ }_{3}^{3}$ Debo aclarar que no es mi intención sostener que ésta es la interpretación correcta en algún sentido trascendente, sino que las herramientas lógicas que he reseñado son inadecuadas para reconstruir el razonamiento de los jueces, en tanto ellos aceptan, como de hecho ocurre en la jurisprudencia actualmente prevaleciente, que ésta es la interpretación correcta. 
Supongamos que una norma establece que cuando se presentan ciertas circunstancias, por ejemplo, que se celebre un contrato de compraventa, se debe pagar un impuesto de $\$ 1.000$, y que otra norma prescribe que cuando acaecen otras circunstancias lógicamente independientes de las primeras, por ejemplo, que se obtengan ingresos extraordinarios, se debe pagar un impuesto de $\$ 1.000$. Parece natural pensar que en el caso en que concurren ambos tipos de circunstancias, esto es, que la celebración del contrato de compraventa a la vez genera ingresos extraordinarios, hay que pagar $\$ 2.000$, es decir, $\$ 1.000$ por el concepto de «compraventa» y $\$ 1.000$ por el concepto de «ingresos extraordinarios». Sin embargo, si analizamos la cuestión con las herramientas lógicas que hemos visto hasta el momento, o con el aparato conceptual de Alchourrón y Bulygin (1974), la conclusión será muy distinta. En efecto, la primera norma permite inferir, a través de la ley de refuerzo del antecedente $-\mathrm{o}$ de las relaciones entre universos de casos de distintos niveles-, que si se celebra un contrato de compraventa y se obtienen ingresos extraordinarios, se deben pagar \$1.000. Lo mismo se puede inferir de la segunda norma. Por tanto, diríamos que nos encontramos con una redundancia normativa, porque ambas normas implican, para el caso en que concurren ambas circunstancias, la misma solución normativa, a saber, la obligación de pagar $\$ 1.000$. Este resultado es, a mi entender, claramente contrario a los criterios de interpretación normalmente aceptados por los juristas.

\section{Empleo de la lógica cuantificacional}

Para analizar adecuadamente el problema no es suficiente el aparato de la lógica proposicional. El empleo de lógica cuantificacional, en cambio, permite detectar dónde reside la dificultad y cuál es el camino para la solución, como intentaré mostrar a continuación.

La interpretación de las fórmulas de la lógica cuantificacional requiere, entre otras cosas, que se defina el universo de entidades del que tomarán sus valores las variables individuales. Si bien esta cuestión es sumamente problemática en el ámbito de la lógica deóntica, para los fines de este trabajo supondré, siguiendo una propuesta de Hintikka (1971), que las entidades en cuestión son acciones individuales.

Utilizaré las letras «X» e «y» como variables de acciones individuales; «a» $\mathrm{y} \ll b »$, como constantes individuales; «F» representará el predicado monádico: «(_) ejemplifica una actividad onerosa habitual realizada en el territorio de la provincia de Buenos Aires»; «G» representará el predicado monádico: «(_)es una acción de transitar de una provincia a otra» $\mathrm{y}$ «H», el predicado diádico: «(_) es una acción de pagar impuestos por la realización 
de $\left(\_\right){ }^{4}$. Con estos elementos, parece que podríamos representar la estructura de las normas que estamos considerando de la siguiente manera:

(1) Norma 1: $(\mathrm{x})(\mathrm{Fx} \rightarrow \mathrm{O}(\mathrm{Ey})(\mathrm{Hyx}))^{5}$

(2) Norma $2:(\mathrm{x})(\mathrm{Gx} \rightarrow \mathrm{P}(\mathrm{y})-(\mathrm{Hyx}))^{6}$

De 1 se puede inferir, por ejemplificación universal:

(3) $\mathrm{F} a \rightarrow \mathrm{O}$ (Еy) ( $\mathrm{Hy} a)$

A su vez, de (2) se infiere, en virtud de la misma regla:

(4) $\mathrm{G} b \rightarrow \mathrm{P}(\mathrm{y})-(\mathrm{Hy} b)$

Supongamos que el acto $a$ efectivamente tiene la propiedad F, de modo que es verdad

(5) $\mathrm{Fa}$

y supongamos que $b$, a su vez, es un acto que tiene la propiedad G, por lo que también es verdad

(6) $\mathrm{G} b$

De (3) y (5) se infiere, por modus ponens,

(7) $\mathrm{O}$ (Еy) (Нya)

y de (4) y (6), también por modus ponens

(8) $\mathrm{P}(\mathrm{y}) \sim(\mathrm{Hy} b)$

Supongamos ahora que $a$ y $b$ son en realidad el mismo acto

(9) $a=b$

La identidad nos autoriza a sustituir $b$ por $a$ en cualquier enunciado sin que se altere el valor de verdad. Por tanto, podemos hacer la sustitución en el enunciado $«(\mathrm{y})\ulcorner(\mathrm{Hy} b) »$ que aparece en $(8)$ a la derecha de $« \mathrm{P} »$ e inferir

(10) $\mathrm{P}(\mathrm{y}) \sim(\mathrm{Hy} a)$

A partir de (7) y (10) obtenemos la conjunción:

(11) $(\mathrm{O}($ Ey) $(\mathrm{Hy} a)) \&(\mathrm{P}(\mathrm{y}) \sim(\mathrm{Hy} a))$

Es evidente que (11) es una contradicción normativa, ya que la fórmula ubicada inmediatamente a la derecha del operador «O» es la negación de la ubicada inmediatamente a la derecha del operador «P», de modo que su forma general es «Op \& $\mathrm{P} r p »$.

La razón por la cual la generalidad de los jueces y juristas no encuentran contradicción alguna parece ser que no conciben a las normas tributarias como enunciados que relacionan simplemente el que acaezcan ciertos

${ }^{4}$ Siguiendo a Frege (1891), represento a los predicados como oraciones incompletas, con lugares vacíos que es adecuado llenar con términos singulares.

${ }^{5}$ Para todo $x$, si $x$ ejemplifica una actividad onerosa habitual realizada en el territorio de la provincia de Buenos Aires, entonces es obligatorio que exista un $y$ tal que $y$ es una acción de pagar impuestos por la realización de $x$.

${ }^{6}$ Para todo $x$, si $x$ es una actividad de transitar de una provincia a otra, entonces está permitido que para todo $y$, no sea el caso que $y$ es una acción de pagar impuestos por la realización de $x$. 
eventos con la obligación de pagar impuestos, o con la permisión de no pagarlos, sino que piensan que la forma en que éstos son presentados o descritos juega un papel esencial. En otras palabras, es necesario dar cuenta de la idea de que un mismo evento puede dar lugar a la aplicación de un impuesto por determinado concepto y a una exención impositiva por otro concepto, y que ello no involucra contradicción en tanto se trate de conceptos diferentes.

En mi opinión, la reconstrucción adecuada del razonamiento que subyace a cualquier interpretación que pueda ser considerada aceptable por los juristas debe apoyarse en la distinción entre contextos extensionales y no extensionales, como veremos a continuación.

\section{Contextos extensionales y no extensionales.}

En la teoría del significado es común distinguir entre la extensión y la intensión de los términos y de las oraciones.

La extensión de un término general es el conjunto de entidades de las que el término puede predicarse con verdad. La intensión puede concebirse como la propiedad expresada por el término.

Las condiciones de identidad son distintas para las extensiones y para las intensiones. Dos términos generales determinan la misma extensión si y sólo si son predicados con verdad exactamente de las mismas entidades. En cambio pueden tener, aun en ese caso, distintas intensiones. Por ejemplo, si todas las cosas azules fueran redondas y todas las cosas redondas fueran azules, los términos «azul» y «redondo» tendrían de hecho la misma extensión, pero sus intensiones serían diferentes: las propiedades de ser azul y de ser redondo respectivamente.

Una dificultad con las intensiones es que no es claro en qué condiciones se puede afirmar que dos términos generales tienen la misma intensión, i.e. expresan la misma propiedad. Sin embargo, para los fines de este trabajo basta con admitir que la identidad de intensiones es condición suficiente para que dos términos sean lógicamente equivalentes. No es indispensable asumir, en cambio, que sea condición necesaria.

Con respecto a los términos singulares -nombres propios y descripciones definidas-, la distinción entre extensión e intensión corresponde aproximadamente a la efectuada por Frege (1892) entre la denotación (Bedeutung) y el sentido (Sinn) del término. La extensión es aquéllo a que el nombre o la descripción definida se refiere -cuando tal cosa existe-. La intensión de un nombre o de una descripción definida es el modo de presentación del objeto. La idea de Frege es que un objeto puede ser identificado de diversas maneras, y cada una de estas maneras de identificarlo puede dar lugar a un nombre propio. Estos nombres propios han de tener diferentes sen- 
tidos, porque no es auto-evidente para un hablante competente del lenguaje que sean nombres del mismo objeto ${ }^{7}$.

Por último, la extensión de una oración suele ser identificada con su valor de verdad, y su intensión, con la proposición expresada o con el sentido de la oración. ${ }^{8}$

Para los fines de este trabajo, es importante destacar:

(a) Para captar la intensión de una expresión sólo es necesario comprender el lenguaje al que esa expresión pertenece. En cambio, en general eso no es suficiente para conocer su extensión. ${ }^{9}$

(b) Que dos expresiones tengan la misma o distinta intensión es una cuestión semántica.

(c) Si dos expresiones tienen la misma intensión, necesariamente tienen la misma extensión.

(d) Si dos expresiones tienen distinta intensión, que tengan igual o distinta extensión es una cuestión fáctica.

Diré que una expresión ocurre extensionalmente en determinado lugar de una oración, y que el contexto es extensional respecto de esa expresión -en ese lugar- si la expresión es intercambiable, en ese lugar, por cualquier otra que tenga la misma extensión sin que se altere el valor de verdad de la oración. En caso contrario, diré que la expresión no ocurre extensionalmente y que el contexto no es extensional respecto de esa expresión en ese lugar. ${ }^{10}$ Adviértase que una expresión puede ocurrir extensionalmente en un

${ }^{7}$ No resulta muy intuitivo hablar del sentido de un nombre propio, pues parece que el nombre se aplica a determinada cosa con total independencia de cualquier propiedad que esa cosa tenga y de cualquier propiedad que el nombre pueda expresar. Por eso, una importante línea filosófica, cuyos representantes más destacados son Mill, Russell y, más recientemente, Kripke, sostiene que los nombres propios genuinos sólo tienen extensión. Russell no considera a los nombres propios del lenguaje ordinario como nombres genuinos, sino como descripciones encubiertas, por lo que deben ser tratados del mismo modo que las descripciones definidas, las cuales son analizadas en términos de variables y predicados. Esto es relevante para el tratamiento de los nombres y descripciones definidas que no nombran nada, pero no necesitamos discutirlo en este trabajo.

${ }^{8}$ Es común que se identifique a la proposición con el sentido de una oración declarativa. Sin embargo, no todos los autores coinciden en esto. En Carnap (1947 y 1956), el criterio de identidad de las proposiciones está basado en la equivalencia lógica: dos oraciones expresan la misma proposición si y sólo si son lógicamente equivalentes. En cambio, la identidad de sentido se basa en lo que denomina «isomorfismo intensional», que es una relación más estricta que la equivalencia lógica. Como consecuencia, en su concepción todas las tautologías expresan la misma proposición, pero pueden tener diferentes sentidos.

${ }^{9}$ Una excepción son las oraciones lógicamente verdaderas o lógicamente falsas.

${ }^{10}$ Esta presentación sólo difiere de la de Carnap (1947 y 1956) porque este autor evita toda referencia expresa a extensiones e intensiones al definir las nociones de «contexto extensional» $\mathrm{y}$ «contexto intensional» (pp. 46 y ss.), pero sí recurre a estas nociones en las explicaciones extrasistemáticas. 
lugar de una oración y no extensionalmente en otro lugar de la misma oración. Por ejemplo, en la oración: «Juan Pablo II eligió llamarse «Juan Pablo II» en homenaje a su predecesor» la primera aparición del nombre «Juan Pablo II» es extensional, ya que puede ser sustituida por «Karol Wojtyla» o cualquier otro nombre o descripción definida que identifique al mismo individuo sin que se altere el valor de verdad de la oración. Ello no ocurre, en cambio, con la segunda aparición.

Un tipo de enunciados que ha sido considerado como un caso típico de contextos no extensionales respecto de los términos singulares que aparecen en ellos lo constituyen los juicios modales.

Esta característica de los contextos regidos por operadores modales puede ilustrarse con un famoso ejemplo de Quine (1962). Tomemos los siguientes enunciados verdaderos:

(1) 9 es necesariamente mayor que 7

(2) el número de los planetas $=9$

Si el numeral «9» ocurriera extensionalmente en (1), podría ser sustituido por «el número de los planetas», que tiene la misma extensión -como indica (2) - sin que se alterara el valor de verdad. Sin embargo, al efectuar la sustitución obtenemos el enunciado falso:

(3) El número de los planetas es necesariamente mayor que 7.

Esto tiene repercusión en el uso de los cuantificadores. Aparentemente, a partir de (1) podríamos inferir, en virtud de la regla de generalización existencial:

(4) (Ex) (x es necesariamente mayor que 7) [hay algo que es necesariamente mayor que 7]

Sin embargo, la oración (4) es extraña. Quine se pregunta: ¿cuál es esa cosa que es necesariamente mayor que 7? ¿Será el número 9, es decir, el número de los planetas? Como es obvio, cualquiera sea el número que mencione, podría también nombrarlo por medio de una descripción definida basada en características que ese número tiene contingentemente. Empero, por otra parte, parece razonable pensar que la cuestión de si una cosa tiene necesariamente una propiedad ha de ser independiente de la forma en que la nombremos.

Este problema parece replantear la antigua discusión sobre si las modalidades son de re o de dicto, es decir, si la necesidad es una propiedad metafísica que tienen las cosas o estados de cosas, o si se predica de los enunciados. En el primer caso, se podría considerar verdadero lo expresado en (4) y aun aceptar como verdadero, bajo cierta interpretación, que el número de los planetas es necesariamente mayor que $7 .{ }^{11}$ Pero esto requiere que

${ }^{11}$ La oración «el número de los planetas es necesariamente mayor que 7» es en realidad ambigua. Puede querer decir: (a) que el número que en el mundo real es nombrado por la descripción definida «el número de los planetas» es mayor que 7 en todo mundo posible, o bien, 
se divorcie la idea de necesidad de la de analiticidad, admitiendo que un estado de cosas puede ser necesario aunque la oración que lo exprese no sea analítica. En cambio, en la concepción de las modalidades como de dicto el enunciado (4) no es admisible: no tiene sentido decir que algo tiene necesariamente la propiedad de ser mayor que 7. Lo que tiene sentido es decir que la oración «9 es mayor que 7» es necesariamente verdadera. Y esto puede ser así y al mismo tiempo ser falso que la oración «el número de los planetas es mayor que 7» sea necesariamente verdadera, porque ambas oraciones tienen distinta intensión -expresan distintas proposiciones- y, mientras la primera es analítica, la segunda no lo es. Los problemas se presentan cuando se mezcla la cuantificación con una concepción de la necesidad basada en la analiticidad. ${ }^{12}$

Quine (1962) sugiere que una vía de solución para poder mantener la cuantificación en los enunciados modales podría consistir en que se manejen en forma totalmente independiente dos universos de discurso, uno formado por lo que llama «objetos extensionales», i.e. las extensiones, y otro por lo que llama «objetos intensionales», i.e. las intensiones, utilizando variables distintas -y también distintos criterios de identidad- para cada uno de estos universos.

\section{La normas como contextos no extensionales}

Cabe preguntarse si es adecuado considerar a las normas como contextos extensionales o como contextos no extensionales respecto de las acciones o circunstancias que se mencionan en ellas y, consiguientemente, si tales acciones o circunstancias han de ser tratadas como objetos extensionales o intensionales.

Para aclarar la pregunta conviene recordar que un mismo conjunto de movimientos corporales puede instanciar a la vez distintas acciones genéricas (p. ej. mover el interruptor de luz, iluminar la habitación, alertar al ladrón, despertar a mi esposa). Si se considera a las acciones individuales como objetos extensionales, estas instanciaciones serán consideradas como

(b) que, en todo mundo posible, el número que en ese mundo sea designado por la expresión «el número de los planetas» es en ese mundo mayor que 7. En la primera interpretación, la referencia queda fijada, y permanece la misma, i.e. el número 9, a través de todos los mundos posibles. De acuerdo con la terminología introducida por Kripke (1972 y 1980) se dirá que la descripción definida «el número de los planetas» se comporta como un designador rígido. En este caso la oración «el número de los planetas es necesariamente mayor que 7» es verdadera, y también lo es la oración (4) del texto. En la segunda interpretación, la referencia de la descripción definida «el número de los planetas» varía de un mundo a otro según cuantos planetas haya en ese mundo. Por tanto, la oración «el número de los planetas es necesariamente mayor que 7» es falsa, y también es falsa la oración (4) del texto.

${ }^{12}$ Una buena explicación de esta cuestión puede encontrarse en Orayen (1995). 
distintas propiedades de la misma acción individual. En cambio, si se las concibe objetos intensionales, se dirá que son acciones diferentes.

En forma más general, en relación a todo tipo de acontecimientos, sean acciones o no, esta diferencia de tratamiento, como objetos intensionales o extensionales, se puede encontrar reflejada en la distinción entre hechos y eventos.

En este sentido, Bennett (1988) señala que tanto los nombres de hechos como los de eventos pueden ser derivados de oraciones, pero, en el caso de los hechos, dos nombres derivados de oraciones que no sean lógicamente equivalentes no pueden ser nombres de un mismo hecho, mientras que ello no ocurre con los nombres de eventos. ${ }^{13}$ Bennett ilustra este punto con el siguiente ejemplo:

De la oración «Bernard viajó una sola vez en su vida a Calais», podemos derivar el nombre de evento:

(e1) su viaje a Calais

y el nombre de hecho:

(h1) el hecho de haber viajado a Calais

$\mathrm{Si}$ ahora agregamos que este viaje lo realizó cruzando a nado el Canal de la Mancha, podemos derivar:

(e2) su cruce a nado del Canal

(h2) el hecho de haber cruzado el Canal a nado

Mientras (e1) y (e2) nombran, según Bennett, el mismo evento, (h1) y (h2) no nombran el mismo hecho. En efecto, su cruce a nado del Canal tiene que ser su viaje a Calais, porque si no fuera así, argumenta este autor, o bien tendría que ser verdad: (a) que su cruce a nado del Canal no fue un viaje a Calais, o bien (b) que no fue su único viaje a Calais. Pero (a) es falso porque, si el cruce a nado lo llevó a Calais, parece claro que fue un viaje a Calais, y (b) también es falso, porque es absurdo sostener que fue a Calais una sola vez en su vida pero hizo más de un viaje a Calais (p. 11).

Por otro lado, Bennet apoya su afirmación de que (h1) y (h2) identifican distintos hechos en que no son intercambiables en explicaciones causales. Por ejemplo, puede ser verdad que Bernard se resfrió por el hecho de haber cruzado a nado el Canal y, a la vez, ser falso que se resfrió por el hecho de haber viajado a Calais. Paralelamente, es posible que yo esté sorprendido por el hecho de que haya viajado a Calais y no lo esté por el hecho de que haya cruzado a nado el Canal.

${ }^{13}$ «... any difference in content between two such fact names -corresponding to any failure of logical equivalence between the parent sentences- makes them names of different facts. If $S_{1}$ is not interdeducible with $S_{2}$, then the fact that $\left[S_{1}\right]$, if there is such a thing, is not the fact that $\left[\mathrm{S}_{2}\right] . »($ p. 9$)$. 
Volvamos ahora a la pregunta inicial de este apartado. Si se tiene en cuenta que la lógica deóntica se desarrolló, al menos en una de sus vertientes más importantes, como una rama de la lógica modal, cabría esperar que el tratamiento de las normas como contextos extensionales presentara dificultades similares a las señaladas por Quine con relación a la lógica modal y, consiguientemente, podría parecer adecuado considerarlas como contextos no extensionales.

Sin embargo, no es evidente que esto pueda ser afirmardo de manera general. Supongamos, por ejemplo, que una norma establece que está prohibido matar. Parece claro que, de acuerdo con esta norma, deberíamos entender que la realización voluntaria de un determinado conjunto de movimientos corporales que tenga como consecuencia la muerte de una persona está prohibida, cualquiera sea la forma en que lo describamos. En otras palabras, el hecho de que podamos identificar ese conjunto de movimientos como una acción de matar, y también como la acción de disparar un revólver, o de apretar un gatillo, o de provocar un estruendo, no hace que lo consideremos prohibido bajo una descripción y no bajo otra. Por el contrario, parece que basta con que haya una descripción de ese conjunto de movimientos bajo la cual está caracterizado como prohibido, es decir, que de hecho tenga las propiedades mencionadas en la norma, para que se considere que está prohibido. Si, en determinadas circunstancias, la acción de contraer el índice de la mano derecha es idéntica a la acción de matar, entonces está prohibido contraer el índice de la mano derecha en esas circunstancias. En términos generales, esto significa que si está prohibida la acción individual $a$ y $a=b$, entonces está prohibida $b$, aun cuando aceptemos un criterio de identidad de acciones que las trate como objetos extensionales, es decir, que admita que dos nombres o descripciones definidas que no sean sinónimos o lógicamente equivalentes pueden identificar la misma acción.

Sin embargo, este tratamiento no parece satisfactorio para abordar el problema que nos ocupó en los apartados II y III.

En efecto, allí obteníamos una contradicción a partir de admitir la identidad entre dos acciones individuales (ap. III, paso 9) que habían sido caracterizadas a través de propiedades lócgicamente independientes: F y G respectivamente. Como vimos, la contradicción, en este caso, no resultaba acorde con las intuiciones prevalecientes de los juristas.

Mostraré ahora cómo, siguiendo la sugerencia de Quine de manejar dos universos distintos, totalmente independientes entre sí, uno de objetos intensionales y otro de objetos extensionales, con diferentes variables para cada universo, la contradicción no se produce.

Para ello reproduciré el argumento desarrollado en el apartado III, manteniendo la interpretación que allí se indicó y el universo de discurso de las 
variables individuales allí empleadas como un universo de objetos extensionales; es decir que continuaré tratando a las acciones individuales como objetos extensionales. Sin embargo, agregaré las letras $\lambda$ y $\phi$ como variables, y $\alpha$ y $\beta$ como constantes, que se interpretan en otro universo de discurso, integrado por objetos intensionales, a los que llamaré «atributos». Las condiciones de identidad de estos objetos no son del todo claras pero asumiré que la equivalencia lógica de dos nombres o descripciones definidas es condición necesaria para que sean nombres de la misma entidad.

Con este agregado, analizaré la estructura de las normas 1 y 2 del apartado III de la siguiente manera:

(1) Norma 1: (x) $(\lambda)((\mathrm{Fx} \& \lambda=$ el atributo de $\mathrm{x}$ de ser $\mathrm{F}) \rightarrow \mathrm{O}(\mathrm{Ey})(\mathrm{Hy} \lambda))$

(2) Norma 2: $(\mathrm{x})(\phi)((\mathrm{Gx} \& \phi=$ el atributo de $\mathrm{x}$ de ser $\mathrm{G}) \rightarrow \mathrm{P}(\mathrm{y})\ulcorner(\mathrm{Hy} \phi))$

(3) (Fa \& $\alpha=$ el atributo de $a$ de ser F) $\rightarrow \mathrm{O}$ (Ey) (Hy $\alpha)-$ de 1 por ejemplificación uiversal

(4) ( Gb \& $\beta=$ el atributo de $b$ de ser $\mathrm{G}) \rightarrow \mathrm{P}(\mathrm{y}) \sim(\mathrm{Hy} ß)-$ de 2 por ejemplificación universal

(5) Fa $\& \alpha=$ el atributo de $a$ de ser F -hipótesis

(6) $\mathrm{Gb} \& \mathrm{~B}=\mathrm{el}$ atributo de $b$ de ser $\mathrm{G}$-hipótesis

(7) $\mathrm{O}$ (Ey) $(\mathrm{Hy \alpha})-$ de 3 y 5 por modus ponens

(8) $\mathrm{P}(\mathrm{y})-(\mathrm{Hyß})-\mathrm{de} 4$ y 6 por modus ponens

(9) $a=b$

Como podermos observar, no es posible continuar, a partir de aquí, con el razonamiento que nos llevó a obtener una contradicción. En efecto, aun admitiendo la identidad de las acciones $a$ y $b$, esto no nos permite efectuar la sustitución que antes se había hecho en (8), porque ahora no figura $b$ sino $\beta$ en esa línea y, por cierto, la identidad entre $a$ y $b$ no implica la identidad entre $\alpha$ y $\beta$, pues estos últimos, al ser objetos intensionales, tienen condiciones de identidad más estrictas. Esto parece reflejar de forma más adecuada el modo de razonar de los juristas al interpretar normas que establecen obligaciones y exenciones impositivas.

\section{Conclusión}

En suma, el análisis llevado a cabo en este trabajo pretende mostrar:

(i) Que la reconstrucción adecuada de ciertas formas de razonamiento jurídico requiere la utilización de las herramientas de la lógica cuantificacional.

(ii) Que la utilización de esta herramienta pone de manifiesto que existen graves dificultades para determinar el tipo de entidades que integran el universo de discurso de las variables.

(iii) Que una de esas dificultades reside en que, mientras en algunos contextos parece adecuado concebir a esas entidades como objetos extensiona- 
les, a la manera de eventos, en otros resulta más adecuado concebirlas como objetos intensionales, lo que se acerca más a la forma en que son tratados los hechos.

\section{Referencias bibliográficas}

Alchourrón, Carlos E. y Bulygin, Eugenio (1974): Introducción a la metodología de las ciencias jurídicas y sociales, Buenos Aires, Astrea.

Alchourrón, Carlos E. (1991): «Condicionalidad y la representación de las normas jurídicas», en Alchourrón, Carlos E. y Bulygin, Eugenio: Análisis lógico y Derecho, Madrid, Centro de Estudios Constitucionales.

Bennett, Jonathan (1988): Events and their Names, New York, Oxford University Press.

Carnap, Rudolf (1947 y 1956): Meaning and Necessity, Chicago, The University of Chicago Press.

Frege, Gottlob (1891): «Función y concepto». Traducción al castellano publicada en Frege, Gottlob: Escritos lógico-semánticos, Madrid, Tecnos, 1974.

Frege, Gottlob (1892): «Sobre el sentido y la denotación». Traducción al castellano publicada en Simpson, Thomas Moro (comp.): Semántica filosófica: problemas y discusiones, Buenos Aires, Siglo XXI, 1973.

Hintikka, Jaakko (1971): «Some Main Problems in Deontic Logic», en Hilpinen, Risto (ed.): Deontic Logic: Introductory and Systematic Readings, Dordrecht, D. Reidel, 1971.

Kripke, Saul (1972 y 1980): Naming and Necessity, Oxford, Blackwell.

Orayen, Raúl (1995): «Lógica modal», en Alchourrón, Carlos E., Méndez, José M. y Orayen, Raúl (eds.): Lógica (Enciclopedia Iberoamericana de Filosofía, volumen 7), Madrid, Ed. Trotta y C.S.I.C., 1995.

Quine, Willard V.O. (1962): «Referencia y modalidad», en Quine, Willard V.O. Desde un punto de vista lógico, Barcelona, Ariel. 
$\triangle \quad$ DOXA 22 (1999) 